\title{
The preliminary analysis on effectiveness of the Korean mentalization based treatment for patients with borderline personality disorder
}

Jeong-Ho Seok ${ }^{1,2}$, Jong-Sun Kim², Min-Jea Kim ${ }^{1.2}$, Hae-In Park², Wook-jin Oh², Sun-Woo Choi ${ }^{2}$

2Institute of Behavioral Science in Medicine, Yonsei University College of Medicine, Seoul, Republic of Korea, Korea

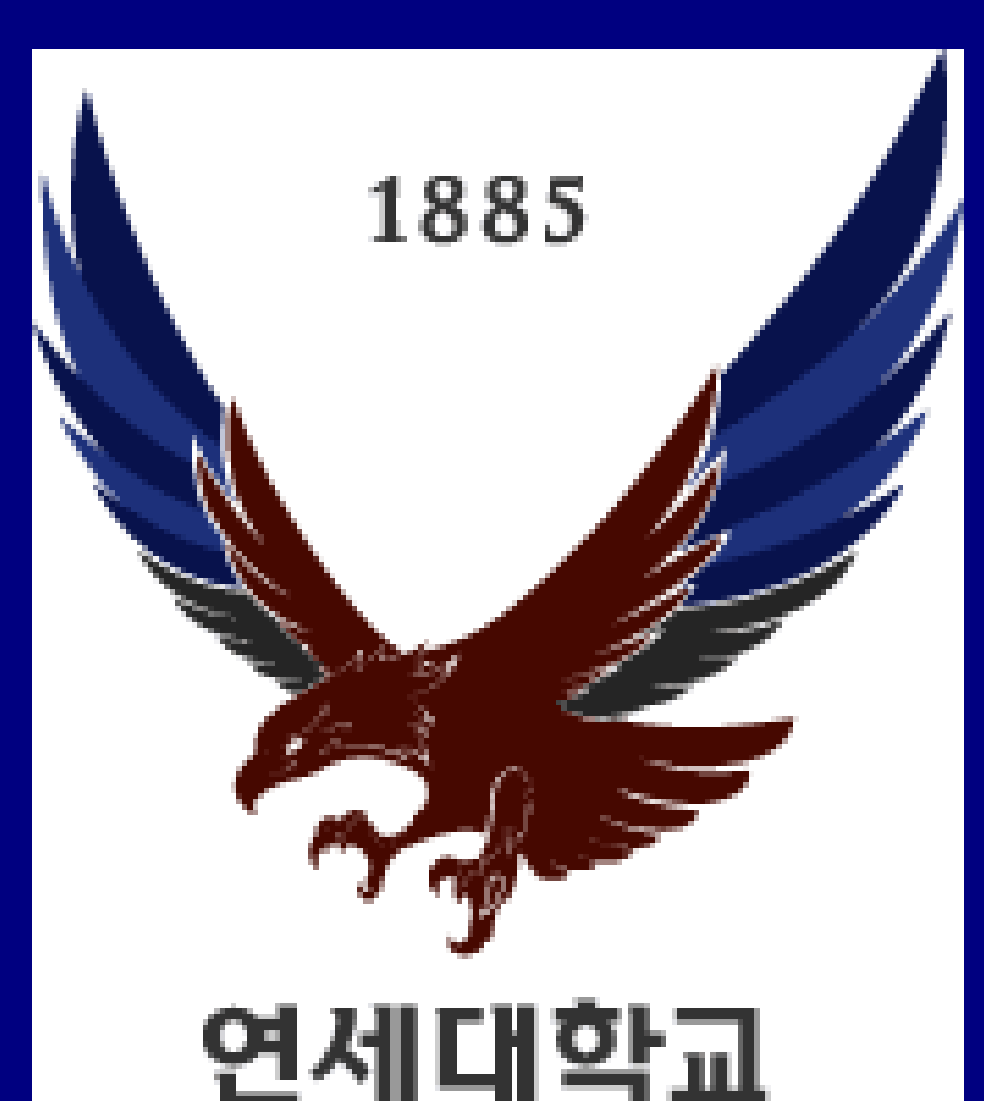

Corresponding author : Jeong-Ho Seok, MD, PhD, e-mail : johnstein@yuhs.ac

연세대학교

\section{Objectives}

Borderline personality disorder (BPD) is characterized by chronic instability in emotion, interpersonal relationships, and self-image. The evidence-based psychotherapeutic approach is recommended as a treatment of choice in the clinical guidelines for BPD. To date, BPD treatment in Korea has mainly consisted of symptom management, crisis intervention based on medication treatment and individual psychotherapy.

In Korea, there is few investigation on the effectiveness group psychotherapy programs for patients with BPD. The mentalization based treatment (MBT) developed in the UK has been modified to Korean culture and a preliminary analysis of its effect was conducted.

\section{METHODS}

\section{Participants}

Patients who were diagnosed as BPD were included in this study. The subjects were assessed about clinical characteristics including personality trait, depressive symptom, mentalization ability, and so on before and after MBT treatment. A total of 62 patients who had completed pre-treatment assessment were analyzed for their clinical characteristics. The analysis of treatment effects was based on data from 21 patients who participated in the treatment group for at least 6 months.

\section{Assessments}

[Baseline]

- Personality Assessment Inventory - Borderline Features Scale (PAI-BOR - Korean version of Quick Inventory of Depressive Symptomatology-Self-Report (K-QIDS-SR)

- Attachment type : Experiences in Close Relationship Questionnaire-Korean (ECR-K) Korean Resilience Questionnaire (KRQ)

- Personality profile : Temperament and Character Inventory (TCI

- Mentalization Questionnaire (MZQ)

- Interpersonal Competence Questionnaire (ICQ

[6-month treatment]

K-QIDS-SR; PAI-BOR; ECR; MZQ; ICQ

[12-month treatment]

- K-QIDS-SR; PAI-BOR; ECR; KRQ; TCI; MZQ; ICQ

\section{Statistical Analysis}

Kolmogorov-Smironov test for normal distribution, paired t-test / Wilcoxon signed rank test were applied to compare changes from baseline clinical features after MBT treatment. Statistical significance level : $p<0.05$

Ethical review and Informed consent

This study was ethically reviewed and approved by our institution and the subjects were given written consent after explaining the study.

\section{î́SULTS}

\section{Demographic characteristics}

Table 1. Demographic characteristics of patients at time of enrollment

\begin{tabular}{|c|c|c|c|}
\hline Variable & $\mathrm{n}(\%)$ & Variable & $n(\%)$ \\
\hline Sex & & Occupation & \\
\hline Male & $11(17.7)$ & Full-time position & $12(19.4)$ \\
\hline Female & $51(82.3)$ & Contingent job & $6(9.7)$ \\
\hline Age & & Student & $15(24.2)$ \\
\hline 19 & $3(4.8)$ & Homemaker & $4(6.5)$ \\
\hline $20 \mathrm{~s}$ & $38(61.3)$ & Inoccupation & $18(29.0)$ \\
\hline $30 \mathrm{~s}$ & $15(24.2)$ & etc & $7(11.3)$ \\
\hline $40 \mathrm{~s}$ & $6(9.7)$ & Family income & \\
\hline Educational background & & Less than 10 million-won & $7(11.3)$ \\
\hline High school graduate & $4(6.5)$ & 10 20 million-won & $5(8.1)$ \\
\hline $\begin{array}{l}\text { Junior college } \\
\text { attendance/graduation }\end{array}$ & $10(16.1)$ & 20 30 million-won & $5(8.1)$ \\
\hline $\begin{array}{l}\text { University } \\
\text { attendance/graduation }\end{array}$ & $42(67.7)$ & 30 40 million-won & $4(6.5)$ \\
\hline $\begin{array}{l}\text { Graduate school } \\
\text { attendance/graduation }\end{array}$ & $6(9.7)$ & 40 50 million-won & $6(9.7)$ \\
\hline Marital status & & 50 60 million-won & $2(3.2)$ \\
\hline Single & $45(72.6)$ & More than 60 million-won & $17(27.4)$ \\
\hline Married & $3(4.8)$ & Do not know & $16(25.8)$ \\
\hline Cohabitation & 2(3.2) & & \\
\hline Divorced & $5(8.1)$ & & \\
\hline etc & $7(11.3)$ & & \\
\hline
\end{tabular}

\section{RIJSULTS}

Borderline personality features was positive correlated with depressive symptom, anxious attachment, avoidant attachment, novelty seeking trait, and showed a negative correlation with self-directedness. After 12-month treatment, depressive symptom and self-disclosure score are significantly improved and self-directedness, cooperativeness factor scores of the temperament and character inventory and selfregulation ability score of the resilience scale are also significantly improved.

Table 2. Changes of psychological characteristics after 12-month treatmen

\begin{tabular}{|c|c|c|c|c|c|}
\hline & Baseline & 12 month & \multirow{2}{*}{$t / Z^{a}$} & \multirow{2}{*}{$p$} \\
\hline & & $\operatorname{Mean}(S D)$ & Mean(SD) & & \\
\hline & $\mathrm{K}$-QIDS-SR & $15.38(9.02)$ & $9.53(6.85)$ & 2.60 & .023 \\
\hline & PAI-BOR & $37.31(13.96)$ & $32.15(12.95)$ & 1.43 & 177 \\
\hline \multirow{2}{*}{ ECR } & Avoidance attachment & $70.46(15.22)$ & $68.62(16.05)$ & .368 & .719 \\
\hline & Anxiety attachment & 79.69(19.97) & 76.46(19.98) & .84 & .416 \\
\hline \multirow{3}{*}{$\mathrm{KRQ}-53$} & Self-regulation & $49.83(10.06)$ & $58.5(14.75)$ & $-2.02^{a}$ & .043 \\
\hline & Interpersonal relationship & $52.33(12.52)$ & $60.0(12.38)$ & $-1.89^{a}$ & .058 \\
\hline & positiveness & $49.0(10.07)$ & $54.67(16.06)$ & $-1.08^{a}$ & .279 \\
\hline \multirow{4}{*}{ MZQ } & Refusing self-reflection & $12(2.45)$ & $11.29(2.43)$ & 1.109 & .310 \\
\hline & Emotional awareness & 13.57(3.31) & $12.43(5.09)$ & 0.834 & .436 \\
\hline & Psychic equivalence & $14.57(2.70)$ & 13.71(3.35) & 1.114 & .308 \\
\hline & Regulation of affect & $11.43(2.70)$ & $10(3.32)$ & 1.594 & .162 \\
\hline \multirow{4}{*}{ ICQ } & Building rapport & $9.86(1.68)$ & $9.86(3.72)$ & $-.135^{a}$ & .892 \\
\hline & Self-assertiveness & $8.57(3.21)$ & $8.57(4.08)$ & $-.106^{a}$ & .915 \\
\hline & Self-disclosure & $4.86(1.57)$ & $6.14(2.19)$ & $-1.983^{\mathrm{a}}$ & .047 \\
\hline & $\begin{array}{l}\text { Empathy and } \\
\text { understanding others }\end{array}$ & 11.86(1.95) & $11.86(2.12)$ & $-.319^{a}$ & .750 \\
\hline
\end{tabular}

Wilcoxon rank-sum test was done for non-parametric analysis

Abbreviations $>$ K-QIDS-SR = Korean version of Quick Inventory of Depressive Symptomatology Self-Report, PAI-BOR = Personality Assessment Inventory-Borderline Features Scale, KRQ-53= Korean Resilience Quotient test-53, ICQ =Interpersonal Competence Questionnaire

\section{Personality proffle change in TCI}

There was a significant improvement in character factor scores but no significant changes in temperament factor scores after 12-month MBT.

Table 3. Changes in temperament and character factors after 12-month treatment

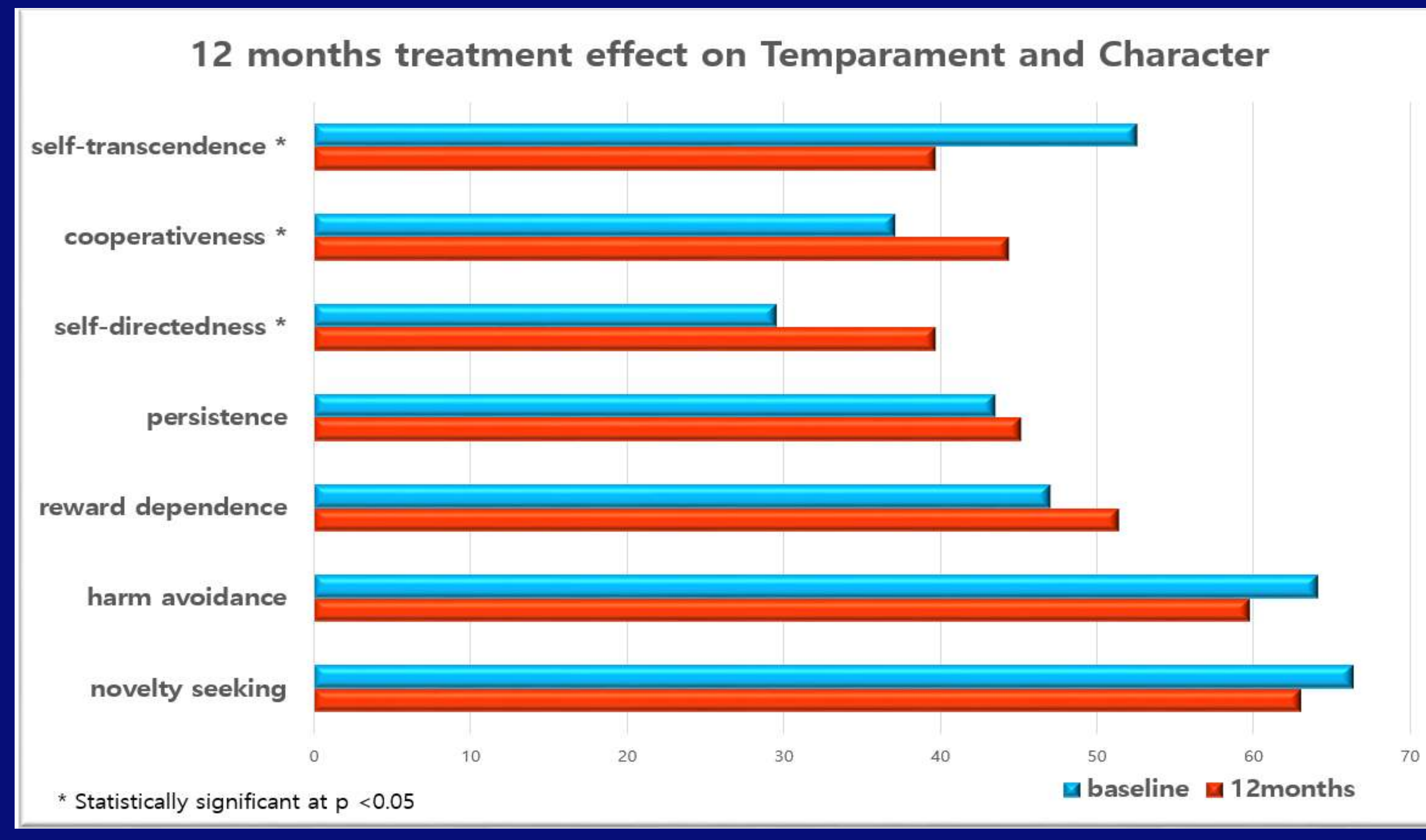

GONGLUSION

This study suggests that Korean MBT can improve depressive symptoms and induce positive changes in personality functioning. Although the number of people who have continued MBT over 12 months is very small due to high drop-out rate, there is an important meaning that this study is the first quantitative study on the effect of Korean MBT for BPD patients.

\section{Refierence}

Bateman, AW., \& Fonagy, P. Mentalization-Based Treatment for Personality Disorders - A Practica Guide, Oxford University Press, 2016 Bateman, AW., \& Fonagy, P. (Eds.). Handbook of mentalizing in mental health practice. Arlington, VA, US: American Psychiatric Publishing, Inc.;2012. Cloninger, CR., Przybeck, TR., Svrakic, DM., \& Wetzel, RD. The Temperament and Character Inventory (TCI): A guide to its development and use. Washington, DC: Center for Psychobiology of Personality, Washington University; 1994 